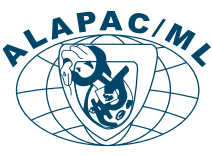

doi: $10.35366 / 93849$
Patología Clínica

y medicina de laboratorio

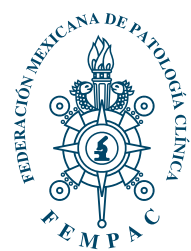

Palabras clave: Anemia, clasificación de anemias, hemoglobina, citometría hemática.

Keywords: Anemia, classification of anemia, hemoglobin, blood cytometry.

* Departamento de Laboratorio Clínico.

‡ Departamento de Oncohematología.

$\S$ Unidad de Trasplante de Médula Ósea.

"l Subdirección de Servicios Auxiliares de Diagnóstico.

Hospital Infantil de México «Federico Gómez».

Correspondencia: Dra. Briceida López Martínez

Subdirección de

Servicios Auxiliares

de Diagnóstico,

Hospital Infantil de

México «Federico

Gómez».

Dr. Márquez Núm. 162,

Col. Doctores, 06720,

Alcaldía Cuauhtémoc, CDMX.

Tel: 5552289917,

ext. 9022

E-mail:

brisalopezmtz@gmail.

com

Recibido:

07/02/2020

Aceptado:

05/03/2020

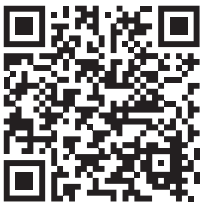

\title{
Frecuencia de anemias en pacientes pediátricos con enfermedades oncológicas
}

\author{
Frequency of anemias in pediatric patients with oncological diseases
}

\author{
Parra Ortega Israel,* Alcaraz Ramírez Diana Guadalupe,* Saucedo Campos Alberto Daniel, ${ }^{\ddagger}$ \\ García Maldonado Paulina, ${ }^{\S}$ Alamillo Hernández Trinidad,* Pozos Loza Gabriela,* \\ Gaytán Morales Félix, ${ }^{\S}$ López Martínez Briceida"
}

\begin{abstract}
RESUMEN
La anemia se define como una concentración de hemoglobina inferior a $11.0 \mathrm{mg} / \mathrm{dL}$, condición en la que el número de glóbulos rojos o su capacidad de transportar oxígeno en la sangre son insuficientes. Los niveles varían según la edad, el sexo, la altitud y algunas otras circunstancias. En México, la anemia por deficiencia de hierro es un problema de salud, y se presenta con mayor frecuencia en niños y en mujeres en edad fértil. Se incluyeron a 229 pacientes, los cuales fueron estudiados por citometría hemática $(\mathrm{CH})$, en la que se evaluó niveles de hemoglobina, volumen globular medio y hemoglobina corpuscular media para clasificar a las anemias, realizar un estudio estadístico y así determinar la frecuencia de anemia por déficit de hierro. Nuestro instituto, el Hospital Infantil de México «Federico Gómez» (HIMFG), al ser un hospital de tercer nivel, atiende principalmente a pacientes afectados por padecimientos crónicos, oncohematológicos, autoinmunes, hepatopatías crónicas, entre otros, por lo que la anemia por deficiencia de hierro no predomina en nuestra población.
\end{abstract}

\section{ABSTRACT}

Anemia is defined as a hemoglobin concentration below $11.0 \mathrm{mg} / \mathrm{dL}$, this condition in which the number of red blood cells or their ability to transport oxygen into the blood is insufficient, which may vary with age, sex, altitude and others circumstances. In Mexico, iron deficiency anemia remains a health problem and is the most common founded in children and women at childbearing age. In our study 229 patients were included and studied by blood cytometry, hemoglobin levels, mean globular volume and mean corpuscular hemoglobin were evaluated, to classify anemias and perform a statistical study to determine the frequency of iron deficiency anemia. Our institution, Hospital Infantil de México «Federico Gómez" (HIMFG), a third level hospital, mainly attend patients overgoing chronic diseases, hemato-oncological, autoimmune, chronic liver diseases, and so other illness, that's why iron deficiency anemia isn't frequent among our patients.

\section{INTRODUCCIÓN}

\section{Definición}

T a Organización Mundial de la Salud (OMS), \lrcorner define a la anemia en niños como una concentración de hemoglobina inferior a $11.0 \mathrm{~g} /$ dL, condición en la que el número de glóbulos rojos o su capacidad de transportar oxígeno en la sangre son insuficientes, niveles que varían con la edad, el sexo, la altitud y otras circunstancias. ${ }^{1}$

\section{Epidemiología}

De acuerdo con datos de la OMS, la anemia afecta a 1.6 mil millones de personas en todo el mundo y la causa más frecuente es de consulta hematológica, lo cual afecta a $30 \%$ de la población mundial de todas las edades y clases sociales. La prevalencia más alta se encuentra en niños de edad preescolar, mientras que las mujeres en edad fértil presentan una mayor incidencia en comparación con los hombres. ${ }^{2}$

Se han realizado diversos estudios epidemiológicos en diferentes regiones de nuestro país, incluyendo zonas rurales y urbanas, en donde se demuestra una alta prevalencia de anemia por deficiencia de hierro, en algunos estudios se ha evaluado la respuesta a la suplementación de hierro y se ha encontrado una adecuada respuesta al tratamiento. Sin embargo, existen casos en los que la concentración de 
hemoglobina no aumentó a pesar de la suplementación, lo que indica que la anemia puede estar asociada con la deficiencia de otros nutrimentos y no sólo a la deficiencia de hierro. ${ }^{3}$

En México, de acuerdo con la última Encuesta Nacional de Salud y Nutrición 2012 (ENSANUT), se utilizó el valor de hemoglobina $<11.0 \mathrm{~g} / \mathrm{dL}$ para definir anemia y se realizó un estudio en 65,249 individuos, se agruparon por edades que iban desde los 12 meses hasta los cinco años de edad, mujeres embarazadas, no embarazadas y hombres, y se definió anemia de acuerdo con los criterios de la OMS. ${ }^{4}$

La anemia por déficit de hierro se define como el descenso de la concentración de hemoglobina en sangre, secundaria a una disminución de la concentración de hierro en el organismo, ya sea por un aporte insuficiente, un aumento de su consumo o un exceso de pérdida. ${ }^{5}$

La deficiencia de hierro es la causa más frecuente de anemia a nivel mundial: hasta $50 \%$ de los niños menores de cinco años y $25 \%$ de las mujeres adultas padecen deficiencia de hierro. ${ }^{6}$

\section{Etiopatogenia}

Algunos pacientes con anemia no manifiestan síntomas o signos, la presencia o ausencia de manifestaciones clínicas se debe a la velocidad de presentación, la severidad, la edad del paciente y la curva de disociación de oxígeno. ${ }^{5}$

La anemia es el resultado de una o más combinaciones de tres mecanismos básicos: ${ }^{7}$

- Pérdida de sangre, éste es el factor etiológico más frecuente en países desarrollados.

- Disminución de la producción de hematíes, los defectos de producción se caracterizan por una disminución de reticulocitos.

- Una destrucción elevada de hematíes (hemólisis).

Cuando no hay suficientes eritrocitos la concentración de hemoglobina es baja, ${ }^{7}$ como resultado se genera hipoxia en el organismo y tejidos. La capacidad de transporte de oxígeno en la sangre normal es de $1.34 \mathrm{~mL} / \mathrm{g}$ de hemoglobina o $20 \mathrm{~mL}$ de $\mathrm{O}_{2}$ por $100 \mathrm{~mL}$ de sangre. ${ }^{7}$ En consecuencia, para evitar su alteración funcional, la hemoglobina incrementa su capacidad para ceder oxígeno a los tejidos y se genera una redistribución del flujo sanguíneo a los órganos más sensibles a hipoxia, como el cerebro y el miocardio, lo que produce una vasoconstricción periférica y la consiguiente palidez en tegumentos. ${ }^{7,8}$

\section{Clasificación de anemias}

La eritropoyesis normal está relacionada con la hemólisis diaria normal de los eritrocitos. Por esta razón, el mantenimiento de una masa eritrocitaria y de una cifra de hemoglobina dentro de valores normales depende del equilibrio entre la formación de eritrocitos y la cantidad que se destruye diariamente (proceso de eliminación y generación de nuevas células). ${ }^{8}$ Es decir, diariamente ingresan a la sangre periférica una cantidad de eritrocitos que fisiológicamente son destruidos. Cuando este equilibrio se ve afectado se produce un proceso anémico. La anemia, por tanto, puede ocasionarse básicamente por una alteración en la producción («anemias centrales») o bien en la destrucción o pérdidas por hemorragia («anemias periféricas»), o en ambas. Las anemias por defecto en la producción o centrales, también denominadas arregenerativas, se caracterizan por tener reticulocitos bajos. Por el contrario, las anemias periféricas, por destrucción o pérdidas, también Ilamadas regenerativas, cursan con reticulocitos elevados. ${ }^{8}$ La clasificación morfológica tiene una importancia clínica y se basa en los cambios en el tamaño y en el contenido de hemoglobina, esta clasificación está dada por los valores del volumen globular medio (VGM) y la concentración de hemoglobina corpuscular media (HCM) (Tabla 1).

\section{Datos de laboratorio}

La citometría hemática $(\mathrm{CH})$ es el estudio de laboratorio destinado a informar sobre el número y características de las células sanguíneas. La interpretación correcta permite establecer sospechas diagnósticas a cerca de la enfermedad que ocasiona las alteraciones del paciente. La citometría hemática puede dividirse en tres grandes grupos: serie roja, serie blanca y serie trombocitaria. Idealmente la medición de todos los parámetros e índices eritrocitarios debe realizarse empleando contadores de partículas por medio de citometría de flujo. ${ }^{9}$

La hemoglobina $(\mathrm{Hb})$ es el principal componente de los eritrocitos y su función es transportar oxígeno y dióxido de carbono a todo el cuerpo. Este parámetro debe ser el único utilizado para definir si se padece anemia. El volumen globular medio (VGM) es de gran valor en el esclarecimiento de la causa de la anemia, ya que permite saber si una anemia es macrocítica (VGM mayor a los límites normales) o microcítica (VGM menor a los límites normales), y la hemoglobina corpuscular media (HCM) permite establecer si la anemia es hipocrómica, cabe destacar que únicamente se puede clasificar como hipocrómica, puesto que no existe la anemia hipercrómica. ${ }^{9}$ La proporción porcentual de eritrocitos inmaduros recientemente desprovistos de núcleo y que han sido liberados al torrente sanguíneo 
se denominan reticulocitos, los cuales al ser teñidos con tinciones supravitales permiten observar restos de ácidos nucleicos, encontrándose elevados en ciertas anemias. ${ }^{9}$

\section{Objetivo}

Determinar la frecuencia y el tipo de anemia que más predomina en los pacientes pediátricos en el Hospital Infantil de México «Federico Gómez», con base en las pruebas de laboratorio clínico de rutina.

\section{MATERIAL Y MÉTODO}

\section{Periodo de estudio}

En un periodo de tres meses (enero de 2019 a marzo de 2019) se incluyeron a todos los pacientes que acudieron al Departamento de Laboratorio Clínico del Hospital Infantil de México «Federico Gómez» (HIMFG) provenientes de la consulta de Oncología, a quienes se les realizó una citometría hemática como parte del abordaje diagnóstico.

La toma de muestra se realizó con un ayuno mínimo de seis horas y se verificaron los estudios requeridos con la solicitud, al igual que los datos de los pacientes. Se tomó una muestra sanguínea en un tubo con ácido etilendiaminotetraacético (EDTA-K $\mathrm{K}_{2}$ ).

\section{Citometría hemática}

La citometría hemática se realizó utilizando el equipo LH780 (BeckmanCoulter), así como el análisis morfológico.

\section{Preparación del extendido}

Se colocó una gota de sangre periférica de aproximadamente $3 \mathrm{~mm}$ de diámetro con ácido etilendiaminotetraacético $\left(\right.$ EDTA- $\left.\mathrm{K}_{2}\right)$ en un extremo del portaobjetos, con ayuda de otro portaobjetos se sostuvo de manera segura

Tabla 1: Clasificación de las anemias de acuerdo a los valores de los índices eritrocitarios. ${ }^{12,13}$

\begin{tabular}{|c|c|c|}
\hline Clasificación & Valores & Tipos de anemias y posible etiología \\
\hline Macrocítica & VGM $>95 \mathrm{fL}$ & $\begin{array}{l}\text { Anemias megaloblástica } \\
\text { Déficit de vitamina } \mathrm{B}^{12} \\
\text { Déficit de ácido fólico } \\
\text { Alteraciones hereditarias en la síntesis de ADN } \\
\text { Alteraciones en la síntesis de ADN producidas por fármacos } \\
\text { Anemias no megaloblásticas } \\
\text { Eritropoyesis acelerada } \\
\text { Anemias hemolíticas con reticulocitosis } \\
\text { Enfermedad hepática }\end{array}$ \\
\hline Normocítica-normocrómica & $\begin{array}{l}\text { VGM } 80-95 \text { fL } \\
\text { HCM } 27 \text { a } 30 \text { pg }\end{array}$ & $\begin{array}{l}\text { Anemias de enfermedades crónicas (la mayoría) } \\
\text { Anemias hemolíticas } \\
\text { Síndromes mielodisplásicos } \\
\text { Infiltración medular } \\
\text { Etapas tempranas de otras anemias } \\
\text { Anemias aplásicas }\end{array}$ \\
\hline Microcítica, hipocrómica & $\begin{array}{l}\text { VGM }<80 \mathrm{fL} \\
\mathrm{HCM}<27 \mathrm{pg}\end{array}$ & $\begin{array}{l}\text { Alteraciones en el metabolismo del hierro } \\
\text { Anemia ferropénica } \\
\text { Anemia de trastorno crónico } \\
\text { Alteraciones en la síntesis de globina } \\
\text { Síndromes talasémicos } \\
\text { Alteraciones en la síntesis de porfirinas y grupo hemo: anemia sideroblástica } \\
\text { Alteraciones en el metabolismo de la vitamina B6 } \\
\text { Intoxicación por plomo } \\
\text { Déficit enzimático }\end{array}$ \\
\hline
\end{tabular}

VGM = Volumen globular medio; HCM = Concentración de hemoglobina corpuscular media . 
ejerciendo una presión muy suave por delante de la gota de sangre en un ángulo de 35-45 grados respecto al otro portaobjetos. Se deslizó hacia atrás para tener contacto con la gota de sangre y posteriormente se realizó el extendido con suavidad y rapidez hacia el otro extremo del portaobjetos. Se dejó secar el extendido al aire libre y posteriormente se realizó la tinción con colorante Wright.

\section{Tinción del extendido con colorante Wright}

Fundamento de la tinción de Wright. La tinción de Wright se utiliza con frecuencia para frotis de sangre periférica, contiene eosina y azul de metileno, por lo que se denominan tinciones policrómicas. ${ }^{10,11}$ Las células se fijan sobre el portaobjetos con metanol. Las reacciones de la tinción dependen del $\mathrm{pH}$; la tinción de los componentes celulares sucede cuando se agrega una solución amortiguadora ( $\mathrm{pH}$ 6.4) a la tinción. El azul de metileno es básico y tiñe de azul a los componentes ácidos como el ácido ribonucleico (ARN), la eosina es ácida y tiñe de rojo los componentes básicos como la hemoglobina o los gránulos eosinófilos. Los neutrófilos poseen gránulos citoplasmáticos que tienen $\mathrm{pH}$ neutro y admiten algunas características de ambas tinciones. ${ }^{10}$

Una vez secos los extendidos se metieron a teñir al equipo Aerospray Hematology $\operatorname{Pro}^{\circledR}$ (un teñidor automático de portaobjetos con un ciclo de tinción rápido y con capacidad de alto rendimiento). Posteriormente se realizó el análisis morfológico de cada caso.

\section{Análisis morfológico}

Se comenzó el análisis de frotis sanguíneo con un barrido del portaobjetos con el objetivo 40x para que se determinara la calidad general del frotis, incluida la distribución anormal de los eritrocitos. El paso siguiente fue colocar una gota de aceite de inmersión y se cambió el objetivo a 100x. Posteriormente se buscó un área en el frotis en la cual los eritrocitos se encontraran uniformemente distribuidos y se realizó el conteo diferencial de modo sistemático; para ello se utilizó un recorrido en patrón guarda griega de leucocitos y el recuento normal de eritrocitos, y de esta manera se observó si existía anisocitosis, o variaciones del tamaño, color o forma de los eritrocitos.

\section{RESULTADOS}

\section{Identificación de pacientes anémicos}

Los pacientes incluidos durante los tres meses fueron agrupados por género y edad, los cuales iban desde un mes de edad hasta los 18 años. Para el análisis de la frecuencia y tipo de anemia se excluyeron a todos los pacientes menores a un año, teniendo al final un total de 229 pacientes, y se definió anemia de acuerdo con los criterios de la OMS (Tabla 2).

Se observó que de la muestra de 229 pacientes, 147 pacientes (64\%) presentaban anemia y sólo 82 pacientes (36\%) no la presentaban. Las proporciones entre sexos fueron significativamente diferentes: en cuanto a las pacientes femeninas, 105 que corresponde al $71 \%$ presentaron anemia, mientras que para los pacientes masculinos se encontraron menos casos con 42 pacientes (29\%).

Como se mencionó anteriormente, los pacientes incluidos fueron pacientes del Departamento de Oncohematología de nuestro instituto, por lo que a continuación se listarán los casos nuevos reportados de patologías oncológicas diagnosticadas durante el año 2019 (Tabla 3).

La distribución de los valores de hemoglobina se encuentra en las Figuras 1 y 2, para pacientes femeninos y masculinos, respectivamente.

Se puede observar en la Tabla 4 que el intervalo de edad en el que se encontraron más casos de anemia fue en pacientes de uno a cuatro años, sumando un total de 98 pacientes $(66.6 \%)$.

\section{Clasificación diagnóstica de anemias en pacientes estudiados}

La clasificación de las anemias con base en el VGM se describe en la Tabla 5 y Figura 3.

Como se muestra en la Tabla 6, de acuerdo con el valor de hemoglobina corpuscular media se observó que 38 pacientes $(40 \%)$ tenían un valor de HCM menor a 27 pg, lo cual indica que los eritrocitos estaban hipocrómicos, es decir, contenían una concentración de hemoglobina inferior al valor de referencia. Por otro lado, 80 pacientes

\begin{tabular}{|c|c|c|}
\hline \multicolumn{3}{|c|}{$\begin{array}{c}\text { Tabla 2: Prevalencia de anemia con base } \\
\text { en la concentración de (Hb) de acuerdo } \\
\text { con los criterios de la OMS. }{ }^{4}\end{array}$} \\
\hline Grupo de población & $\begin{array}{c}\text { Grupo de } \\
\text { edad }\end{array}$ & $\begin{array}{c}\text { Concentración } \\
\text { de } \mathrm{Hb}(\mathrm{g} / \mathrm{dL})\end{array}$ \\
\hline Preescolares & $\begin{array}{l}12 \text { a } 59 \\
\text { meses }\end{array}$ & $<11$ \\
\hline Escolares & 5 a 11 años & $<11.5$ \\
\hline $\begin{array}{l}\text { Mujeres no } \\
\text { embarazadas }\end{array}$ & $\begin{array}{l}12 \text { años y } \\
\text { más }\end{array}$ & $<12$ \\
\hline Mujeres embarazadas & 12 a 49 años & $<11$ \\
\hline Hombres & $\begin{array}{l}12 \text { años y } \\
\text { más }\end{array}$ & $<13$ \\
\hline
\end{tabular}




\section{Tabla 3: Casos nuevos reportados de} patologías oncológicas en el año 2019, en el HIMFG.

\begin{tabular}{lcc} 
Tipo de patología oncológica & $\mathrm{n}$ & $\%$ \\
\hline Leucemia linfoblástica aguda & 50 & 20.74 \\
Leucemia mieloide aguda & 17 & 7.05 \\
Linfoma no Hodgkin & 15 & 6.22 \\
Tumor germinal & 13 & 5.39 \\
Sarcoma de Ewing & 13 & 5.29 \\
Tumor de Wilms & 10 & 4.10 \\
Osteosarcoma & 10 & 4.10 \\
Glioma & 10 & 4.10 \\
Astrocitoma & 10 & 4.10 \\
Histiocitosis células Langerhans & 9 & 3.73 \\
Sarcoma & 8 & 3.31 \\
Hepatoblastoma & 8 & 3.31 \\
Meduloblastoma & 7 & 2.90 \\
Ependimoma & 7 & 2.90 \\
Teratoma & 6 & 2.48 \\
Linfohistiocitosis hemofagocítica & 6 & 2.48 \\
Hepatoblastoma & 6 & 2.48 \\
Linfoma Hodgkin & 6 & 2.40 \\
Neuroblastoma & 5 & 2.07 \\
Papilar tiroides & 4 & 1.65 \\
Retinoblastoma & 3 & 1.20 \\
Rabdomiosarcoma & 3 & 1.20 \\
Ganglioma & 3 & 1.20 \\
Disgerminoma & 3 & 1.20 \\
Tumor neuroectodérmico & 2 & 0.82 \\
Tumor papilar pineal & 1 & 0.41 \\
Hamartoma & 1 & 0.41 \\
GIST (tumor del estroma gastrointestinal) & 0.41 \\
Craneofaringioma & 1 & 0.41 \\
Cáncer testicular & 0.41 \\
Leucemia granulocítica crónica & 0.41 \\
Leucemia del lactante & 0.41 \\
\hline
\end{tabular}

(54\%) se encontraban normocrómicos, y finalmente 29 niños (20\%) se encontraron por arriba de 35 pg (Figura 4).

Una vez identificados todos los pacientes anémicos, es importante tener en cuenta las características mencionadas en la Tabla 1 para conocer la etiología de las anemias.

Además, es preciso mencionar que nuestros pacientes cursan con enfermedades complejas y estados crónicos, ya que somos un hospital de tercer nivel, lo cual hace pertinente un estudio minucioso de la enfermedad de base y la afectación hematológica de manera particular en cada uno de los casos.

\section{DISCUSIÓN}

En México, de acuerdo con la ENSANUT en 2012, los grupos de edades más afectados son los niños en edad preescolar, que van desde los 12 meses a los cinco años de edad, este grupo representó $23.7 \%$ de los pacientes anémicos. Por su parte, en el grupo de niños de cinco a 11 años de edad la prevalencia fue de $16.6 \%$ y para el caso de los adolescentes de 12 a 19 años fue de $11.5 \%$, esto debido al aumento de requerimiento que les demanda su crecimiento físico, lo que hace evidente la necesidad de ingesta de hierro para así poder mantener un adecuado funcionamiento.

De igual forma, se debe mantener un adecuado aporte de hierro a las unidades formadoras de colonias hematopoyéticas, las cuales generan nichos hematopoyéticos

\begin{tabular}{ccc}
\hline \multicolumn{3}{c}{ Tabla 4: Pacientes con valores $<11$ g/dL. } \\
\hline Edad (años) & $n$ & $\%$ \\
\hline >1 a 4 & 98 & 66.6 \\
5 a 11 & 28 & 19.0 \\
12 a 18 & 21 & 12.2 \\
\hline
\end{tabular}

\begin{tabular}{cccr}
\hline \multicolumn{4}{c}{ Tabla 5: Valores de volumen globular medio } \\
que se analizaron de los 147 pacientes con anemia. \\
\hline Valor de VGM & Tipo de anemia & $n$ & $\%$ \\
\hline$<80 \mathrm{fL}$ & Microcítica & 56 & 40 \\
$80-95 \mathrm{fL}$ & Normocítica & 82 & 59 \\
$>95 \mathrm{fL}$ & Macrocítica & 9 & 1 \\
\hline
\end{tabular}

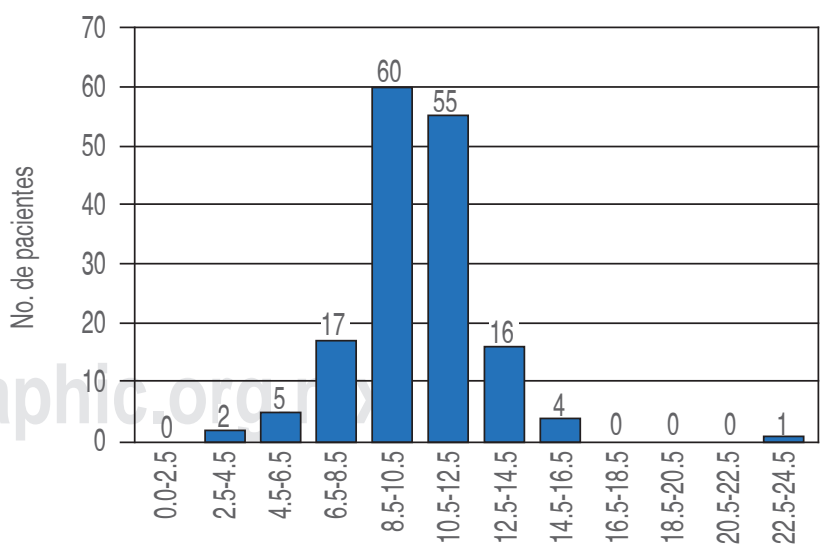

Intervalos del valor de hemoglobina $\mathrm{g} / \mathrm{dL}$

Figura 1: Distribución de los resultados de hemoglobina en pacientes femeninas, estudiados en el HIMFG. 


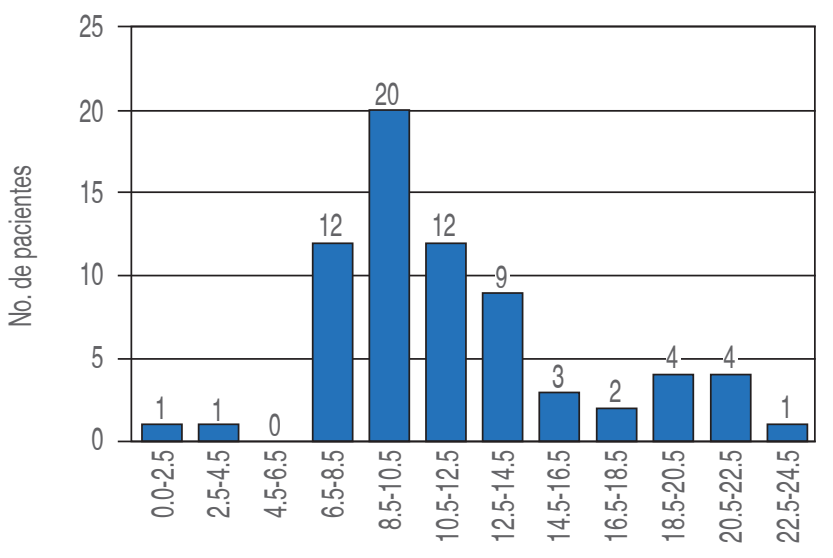

Intervalos del valor de hemoglobina $\mathrm{g} / \mathrm{dL}$

Figura 2: Distribución de los resultados de hemoglobina en pacientes masculinos, estudiados en el HIMFG.

eritroblásticos. ${ }^{14}$ Aproximadamente $30 \%$ de la fuente de hierro se obtiene por el reciclado de la eriptosis y la hemocatéresis en hígado y bazo, mientras que el $70 \%$ restante es obtenido de la dieta.

Por el contrario, durante la edad adulta, este porcentaje se invierte, siendo el 70\% obtenido por el reciclaje de eritrocitos y el $30 \%$ restante obtenido de la ingesta diaria.

En el caso de las mujeres de edad fértil, debido a la pérdida de hierro por la menstruación, la necesidad de hierro ingerido y hierro reciclado varía, pero aproximadamente se mantiene en $50 \%$ según la cantidad, tiempo y duración de la menstruación.

Por otra parte, nuestro organismo está dotado de diversos sitios de reserva de hierro, siendo la reserva en hígado y médula ósea de aproximadamente 1.5 gramos para mujeres y 2 gramos para hombres, lo que asegura un aporte de seis a ocho meses aproximadamente para la eritropoyesis y las funciones fisiológicas, una vez depletado este depósito es cuando se presentan las manifestaciones clínicas de la anemia. ${ }^{15}$

En nuestro país existen diversas publicaciones ${ }^{16-19}$ que describen la frecuencia y las características de los pacientes mexicanos, cabe resaltar que las características geográficas (principalmente la altura sobre el nivel del mar) tienen un efecto importante, ya que valores de hemoglobina que se consideran normales en algunas ciudades son bajos en algunos otros sitios; por lo tanto, estudiar de manera detallada cada caso es una tarea del clínico, considerando la integración de todas las pruebas de laboratorio pertinentes. Otro punto importante es el tratamiento, pues se ha evidenciado que en algunos pacientes resulta ineficiente el mismo y deben realizarse algunas modificaciones, principalmente en las anemias por deficiencia de hierro. ${ }^{19}$ Se ha descrito que en nuestro país la presencia de síndromes anémicos en niños no es infrecuente y que los diagnósticos en la primer línea de atención medica deben ser más eficientes. ${ }^{20-22}$

Es necesario generar más información para que el médico de primer contacto pueda diagnosticar las anemias de manera eficiente, y con apoyo de las diferentes herramientas diagnósticas identifique la causa y establezca un tratamiento oportuno.

En este trabajo inicialmente se evaluaron los índices eritrocitarios para establecer el diagnóstico en los pacientes estudiados. En los casos en los que se diagnosticaron alteraciones hematológicas y que requirieron de un estudio más especializado para identificar la etiología de la anemia se recurrió a la prueba de fragilidad a las soluciones hipotónicas o a la evaluación de las moléculas CD55 y CD59. Dichos pacientes fueron referidos al Servicio de Hematología para un abordaje integral. Con

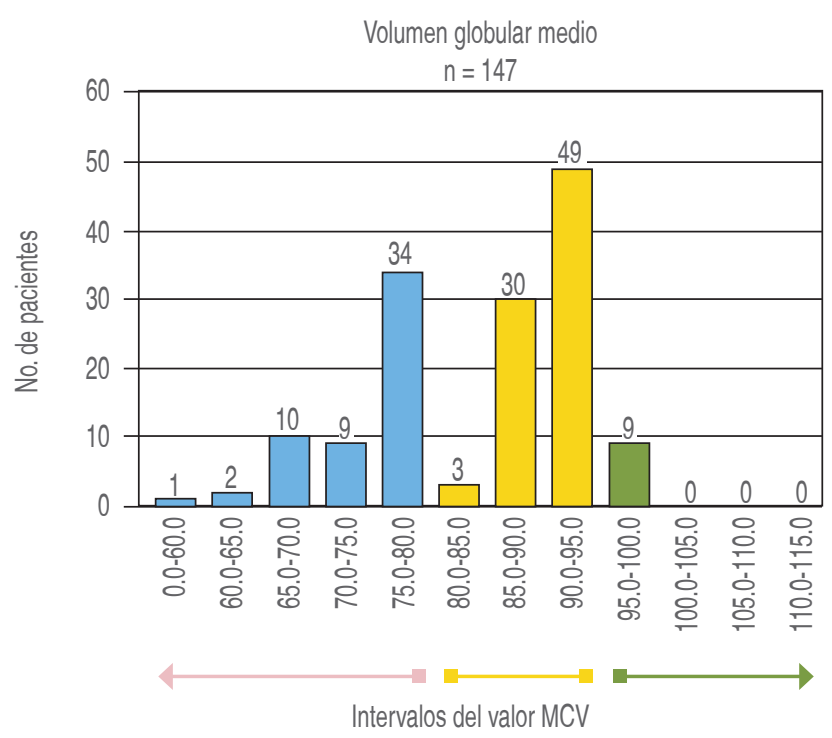

Figura 3: Distribución de resultados del valor VGM, de los 147 pacientes con anemia.

\begin{tabular}{cccc}
$\begin{array}{c}\text { Tabla 6: Valores de hemoglobina corpuscular media } \\
\text { del total de pacientes con anemia. }\end{array}$ \\
\hline Valor & Tipo de anemia & $n$ & $\%$ \\
\hline$<27 \mathrm{pg}$ & Hipocrómica & 38 & 26 \\
27 a $30 \mathrm{pg}$ & Normocrómica & 80 & 54 \\
$>35 \mathrm{pg}$ & N/A & 29 & 20 \\
\hline
\end{tabular}




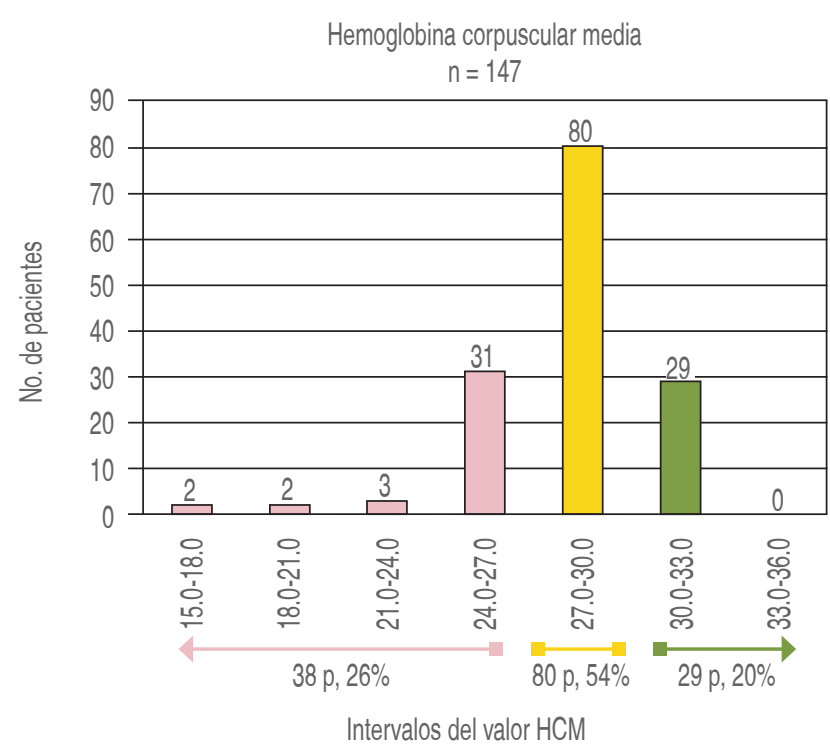

Figura 4: Distribución de resultados del valor de HCM del total de pacientes con anemia.

excepción de esto último, la citometría hemática es el mejor elemento disponible por costo, tiempo del estudio y eficiencia del mismo para el diagnóstico hematológico de primera línea.

En este estudio clasificamos a las anemias con base en los índices eritrocitarios y se observó que las anemias normocíticas-normocrómicas son las más frecuentes, seguidas de las anemias microcíticas-hipocrómicas. Este hallazgo es justificado, ya que los pacientes cursan con enfermedades oncológicas, las cuales afectan significativamente la hematopoyesis.

En algunos casos en los que los pacientes tienen afecciones renales y hepáticas inherentes a su patología, se hace más evidente la presencia de alteraciones hematológicas (morfológicas y bioquímicas), lo que hace esperar que el porcentaje aumente, ya que al padecer enfermedades inflamatorias es el resultado esperado.

Otro factor que puede influir es el enmascaramiento que puede asociarse con las múltiples transfusiones que reciben estos sujetos de manera crónica, lo que obligaría a estudiar más a fondo las poblaciones eritrocitarias de cada paciente, desde el punto de vista morfológico, así como el estudio de parámetros bioquímicos.

Ya es conocido que las anemias normocíticas-normocrómicas tienen una etiología variable, entre las que se destaca el enmascaramiento por las múltiples transfusiones; la segunda destacada es una anemia iatrogénica al tener múltiples eventos de toma de muestra por día, con dosis excesivas de muestra en cada tubo. Otra causa puede ser la disminución en la producción medular que se observa en pacientes oncológicos por efecto directo del tratamiento antineoplásico, en pacientes con insuficiencia renal por falta de generación de eritropoyetina y en los pacientes hipotiroideos.

La frecuencia de pacientes con anemia macrocítica fue del $1 \%$ en el grupo estudiado. Sin embargo es de gran importancia estudiar a fondo las características fisiopatológicas de este grupo de pacientes. Este grupo incluye la macrocitosis por deficiencia de vitamina B12 o ácido fólico y los pacientes portadores de falsas macrocitosis, las cuales usualmente son secundarias a fenómenos regenerativos acelerados, como puede ser en un evento de pérdida excesiva de sangre o fenómenos hemolíticos sostenidos, lo que puede confundirse con otras entidades.

Por ende es necesario realizar en este caso revisión de la morfología para así consolidar el diagnóstico.

Finalmente, al observar nuestros resultados se observa que $20 \%$ de los individuos revisados presenta hipercromía. Este es un tema muy interesante, ya que es controversial, desde el punto de vista de la hematología citomorfológica, en general se considera que la hipercromía no existe; sin embargo, este dato debe ser estudiado, puesto que usualmente las esferocitosis generan este fenómeno. Por lo tanto, se debe ser muy cauteloso en estos casos, considerando que la revisión del frotis y las características morfológicas deben ser realizadas por un experto.

\section{CONCLUSIÓN}

En nuestro instituto, la anemia normocítica-normocrómica asociada con los padecimientos crónicos es la anemia más frecuente que padecen nuestros pacientes con enfermedades oncológicas.

Por tanto, es de suma importancia realizar el diagnóstico oportuno de la anemia, para así brindar un tratamiento efectivo, el cual revertirá la deficiencia, mejorará la respuesta al tratamiento quimioterapéutico, aumentará la tasa de éxito y por ende la supervivencia.

\section{REFERENCIAS}

1. Organización Mundial de la Salud. Concentraciones de hemoglobina para diagnosticar anemia y evaluar su gravedad. Disponible en: https://www.who.int/vmnis/database/anaemia/ anaemia_data_status_t2/es/.

2. Benoist EM. Base de datos mundial sobre la anemia de la OMS. Organización Mundial de la Salud. Worldwide prevalence of anaemia 1993-2005. Ginebra: Organización Mundial de la Salud; 2008.

3. Martínez-Vázquez MA, Murguía-Martínez P. Información de epidemiología. Anemias. Gac Méd Méx. 1998; 134 (49): 495-500.

4. Martínez-Villegas O, Baptista-González HA. Anemia por deficiencia de hierro en niños: un problema de salud nacional. Rev Hematol Mex. 2019; 20 (2): 96-105. 
5. Fuentes-Arderiu X, Castiñeiras MJ, Queraltó JM. Bioquímica clínica y patología molecular. 2a ed. Barcelona: Reverté, S.A.; 1998.

6. Rodak BF. Hematología: fundamentos y aplicaciones clínicas. Buenos Aires: Médica Panamericana; 2004.

7. Hoffbrand AV, Pettit JE. Hematología básica. México: Editorial Limusa, S.A. de C.V.; 1991.

8. Rifkind RA. Hematología clínica. 3a ed. México D.F.: Interamericana S.A. 1986.

9. Ruiz-Argüelles GJ, Ruiz-Delgado GJ. Fundamentos de hematología. 5a ed. México: Médica Panamericana; 2014.

10. Rodak B, Carr J. Atlas de hematología clínica. 5a ed. Ciudad de México: Médica Panamericana; 2017.

11. Sánchez MVJ, García GJL, Velasco PM, Flores HS, Belmont ML, Orozco MJV et al. Consenso Nacional para el Diagnóstico y Tratamiento de la Anemia en la Infancia y en la Adolescencia. Pediatr Mex. 2012; 14 (2): 71-85.

12. Jiménez JM. Pregrado de hematología. Sociedad Española de Hematología y Hematoterapia. 4a ed. Madrid: LUZÁN 5, S.A.; 2017.

13. Kaushansky K, Lichtman M, Prchal JT, Levi MM, Press OW, Burns LJ et al. Williams hematology. 8a ed. México: McGraw-Hill; 2015.

14. Mayani H, Flores-Figueroa E, Pelayo R, Montesinos JJ, FloresGuzmán P, Chávez-González MA. Hematopoyesis. Cancerología. 2007; 2: 95-107.
15. Martínez-Salgado H, Casanueva E, Rivera-Dommarco J, Viteri FE, Bourges-Rodríguez $\mathrm{H}$. La deficiencia de hierro y la anemia en niños mexicanos. Acciones para prevenirlas y corregirlas. Bol Med Hosp Infant Mex. 2008; 65 (2): 86-99.

16. Ruiz-Argüelles GJ. Classification of the dyshemopoietic anemias. Rev Invest Clin. 1983; 35 (1): 1-2.

17. Ruiz-Argüelles GJ. Iron deficiency anemia in low- and middleincome countries. Blood. 3013; 122 (13): 2289-2289.

18. Ruíz-Argüelles GJ. Altitude above sea level as a variable for definition of anemia. Blood. 2006; 108 (6): 2131; author reply 2131-2132.

19. Ruiz-Argüelles GJ, Díaz-Hernández A, Manzano C, Ruiz-Delgado GJ. Ineffectiveness of oral iron hydroxide polymaltose in iron-deficiency anemia. Hematology. 2007; 12 (3): 255-256.

20. López-García YK, Colunga-Pedraza PR, Tarín-Arzaga L, López-Garza MI, Jaime-Pérez JC, Gómez-Almaguer D. Iron deficiency anemia referral to the hematologist. Real-world data from Mexico: the need for targeted teaching in primary care. Hematology. 2018; 23 (9): 658-663.

21. Mujica-Coopman MF, Brito A, López de Romaña D, Ríos-Castillo I, Coris H, Olivares M. Prevalence of Anemia in Latin America and the Caribbean. Food Nutr Bull. 2015; 36 (2 Suppl): S119-S128.

22. Syed S, Addo OY, De la Cruz-Góngora V, Ashour FA, Ziegler TR, Suchdev PS. Determinants of anemia among school-aged children in Mexico, the United States and Colombia. Nutrients. 2016; 8(7). pii: E387. 